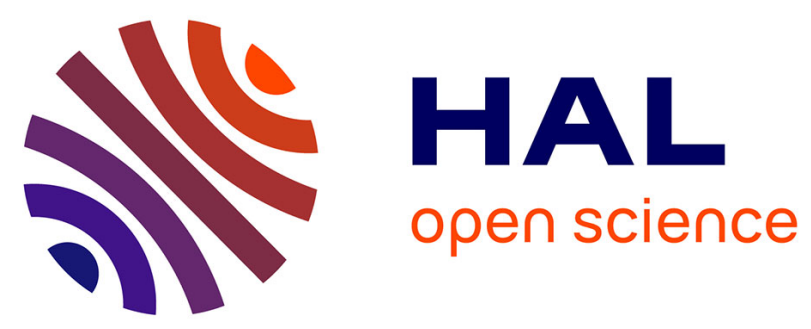

\title{
Les mouvements catholiques pour la paix à travers les papiers du Père Yves de La Brière (s.j.) \\ Bruno Goyet, Emmanuelle Picard
}

\section{To cite this version:}

Bruno Goyet, Emmanuelle Picard. Les mouvements catholiques pour la paix à travers les papiers du Père Yves de La Brière (s.j.). Matériaux pour l'histoire de notre temps, 1993, S'engager pour la paix dans la France de l'entre-deux-guerres, 30, pp.14-20. 10.3406/mat.1993.404086 . hal-00585853

\section{HAL Id: hal-00585853 \\ https://hal.science/hal-00585853}

Submitted on 15 Apr 2011

HAL is a multi-disciplinary open access archive for the deposit and dissemination of scientific research documents, whether they are published or not. The documents may come from teaching and research institutions in France or abroad, or from public or private research centers.
L'archive ouverte pluridisciplinaire HAL, est destinée au dépôt et à la diffusion de documents scientifiques de niveau recherche, publiés ou non, émanant des établissements d'enseignement et de recherche français ou étrangers, des laboratoires publics ou privés.

\section{(ㅇ)(1) $\$$}

Distributed under a Creative Commons Attribution - NonCommercial - NoDerivatives| 4.0 


\section{Persée}

http://legacy.persee.fr

\section{Les mouvements catholiques pour la paix à travers les papiers du Père Yves de la Brière (s.j.)}

\section{Bruno Goyet;Emmanuelle Picard}

Matériaux pour l'histoire de notre temps, Année 1993, Volume 30, Numéro 1

p. $14-20$

Voir l'article en ligne

\section{Avertissement}

L'éditeur du site «PERSEE »- le Ministère de la jeunesse, de l'éducation nationale et de la recherche, Direction de l'enseignement supérieur, Sous-direction des bibliothèques et de la documentation - détient la propriété intellectuelle et les droits d'exploitation. A ce titre il est titulaire des droits d'auteur et du droit sui generis du producteur de bases de données sur ce site conformément à la loi n`98-536 du $1 \mathrm{er} \mathrm{juillet} 1998$ relative aux bases de données.

Les oeuvres reproduites sur le site «PERSEE » sont protégées par les dispositions générales du Code de la propriété intellectuelle.

Droits et devoirs des utilisateurs

Pour un usage strictement privé, la simple reproduction du contenu de ce site est libre.

Pour un usage scientifique ou pédagogique, à des fins de recherches, d'enseignement ou de communication excluant toute exploitation commerciale, la reproduction et la communication au public du contenu de ce site sont autorisées, sous réserve que celles-ci servent d'illustration, ne soient pas substantielles et ne soient pas expressément limitées (plans ou photographies). La mention Le Ministère de la jeunesse, de l'éducation nationale et de la recherche, Direction de l'enseignement supérieur, Sous-direction des bibliothèques et de la documentation sur chaque reproduction tirée du site est obligatoire ainsi que le nom de la revue et- lorsqu'ils sont indiqués - le nom de l'auteur et la référence du document reproduit.

Toute autre reproduction ou communication au public, intégrale ou substantielle du contenu de ce site, par quelque procédé que ce soit, de l'éditeur original de l'oeuvre, de l'auteur et de ses ayants droit.

La reproduction et l'exploitation des photographies et des plans, y compris à des fins commerciales, doivent être autorisés par l'éditeur du site, Le Ministère de la jeunesse, de l'éducation nationale et de la recherche, Direction de l'enseignement supérieur, Sous-direction des bibliothèques et de la documentation (voir http://www.sup.adc.education.fr/bib/ ). La source et les crédits devront toujours être mentionnés. 


\section{Les mouvements catholiques pour la paix à travers les papiers du Père Yves de La Brière (s.j.)}

1. Bulletin de I'Union Catholique d'Etudes Internationales. Cf. paragraphe 2 de cette étude.

2. «Notre programme", p. 3.

3. Goyau est alors président de la "Corporation des publicistes chrétiens". C'est un des

"francs-tireurs" catholiques dans les organisations internationales, telles que le "Groupement universitaire français pour la SDN".

4. Ce sont les cinq niveaux d'analyse du catholicisme que définit R. Aubert, «L'Eglise catholique et le problème de la guerre. Bilan des travaux et état des problèmes «, p. 107, in Les Internationales et le problème de la guerre au vingtième siècle, Ecole française de Rome, Paris, Diffusion De Boccard, 1987.

5. "Conscience internationale et conscience sociale dans les milieux catholiques d'expression française dans l'entredeux-guerres", in Relations Internationales, $n^{\circ} 27$, pp. 361 374.

6. Archives de la Province de France de la Compagnie de Jésus, à Vanves. Fonds Yves de La Brière, cartons 4 (19091927), 5 (1928-1937) et 6 (1938-1939). Désormais JYLB.

7. RP. H. du Passage, Dans le champ de l'Eglise, texte dactylographié de 206 pages, rédigé en 1942 et non publié, conservé à Vanves, Fonds YLB, carton 1.

8. Lettre du 5 IX 1909, Fonds YLB, carton 3

9. J. Prévotat, in Histoire religieuse de la France contemporaine, s.d. de Y.M. Hilaire et G. Cholvy, tome II: 1880-1930, Toulouse, Privat, 1986, p. 299.

10. La Brière, in Construire 1941, p. 149.

11. Fonds YLB, carton 2. C'est nous qui soulignons. e pacifisme des catholiques est une notion difficile à cerner. De quels catholiques et de quel pacifisme veut-on parler? S'il n'est pas question de prendre part à la surenchère idéologique qui ne délivre le brevet de pacifisme qu'aux positions les plus extrêmes, au nom d'une hypothétique pureté doctrinale et militante, privilégiant par là les infimes minorités, exclusives les unes des autres, l'on concevra alors le problème comme celui de l'attitude générale des catholiques en tant que catholiques, donc dans la soumission doctrinale aux autorités hiérarchiques, vis-à-vis de la morale de la guerre et des problèmes internationaux. En tant que tel, le mot de "paix» est trop chargé de sens dans le vocabulaire ecclésiastique, pour que son emploi puisse être innocent. Et la notion d' «Internationale» se heurte au principe même de la catholicité. De quelle autre paix peut-il s'agir si ce n'est de celle du Christ? De quelle autre Internationale, si ce n'est l'Eglise elle-même? Si la devise du pontificat de Pie XI (1922-1939) est : “Pax Christi in regno Christi» que reprend Justice et Paix', le Bulletin Catholique International, dans son premier numéro, le 1er juin 1925, précise

"Nous rejetons l'épithète même de "pacifistes", parce qu'elle recouvre souvent quelque naïveté et qu'elle garde une couleur politique qu'il ne nous convient pas d'adopter "2

Le problème est donc, en premier lieu, un problème de vocabulaire, quand Pie $\mathrm{XI}$, dans l'encyclique Ubi Arcano Dei (1922), affirme que la véritable "societas gentium" n'est pas la Société des Nations laïque, mais la chrétienté médiévale, ou quand le Père de La Brière consacre à celle-ci son cours à I'Institut Catholique de Paris. Le journal de ce dernier fourmille de pointes lancées contre la "phraséologie» pacifiste de gauche ou démocrate-chrétienne. Les pacifistes laïcs sont simplement accusés de messianisme démocratique, dont les formules ne feraient que reprendre subrepticement celles du catholicisme. C'est bien en termes de concurrence que ce jésuite présente très souvent la question du pacifisme, concurrence fondée sur des abus de langage d'après lui. Le catholicisme ne laisserait de place ni au pacifisme, ni à l'internationalisme. Comment alors peut se faire l'articulation du message doctrinal et des exigences de la position internationale du Saint-Siège avec I'habitus national du catholicisme français? Par quelles organisations, spécifiques ou non? Par quels courants?

En l'absence d'un nouveau «Rerum Novarum" consacré aux problèmes internationaux, comme le réclamait Georges Goyau en 1922 dans Papauté et Chrétienté sous Benoit $X V^{3}$, la définition d'une position catholique dans les problèmes internationaux et la conversion de l'opinion catholique aux questions internationales sont l'oeuvre de théologiens, de philosophes et de sociologues, tels les PP. Delos (op), Fessard (sj) et de La Brière (sj), qui vont travailler clergé et peuple des fidèles dans le respect des directives pontificales et en collaboration avec I'Episcopat national ${ }^{4}$. Les Jésuites, en concurrence avec les Dominicains, vont jouer un rôle primordial dans l'élargissement progressif de la doctrine sociale de l'Eglise aux relations internationales, aussi bien aux Etudes et à "l'Action Populaire" du P. Desbuquois qu'aux "Semaines sociales" du Havre (1926), de Marseille (1930) et de Lille (1937).

Il y a dix ans déjà, Jacques Gadille préconisait, pour "cerner les mécanismes et les limites sociologiques de ce mouvement", le "recours aux papiers privés de personnalités marquantes" 5 . Or, le RP. Yves Leroy de La Brière a laissé des archives importantes, en particulier le journal qu'il tint de 1909 à $1939^{6}$, durant toute la période où il eut la charge d'une chronique aux Etudes, et qui permet de cerner l'ambiguiité de la position de l'Eglise et des catholiques face aux problèmes de la paix, de la guerre et du concert des Nations.

\section{Un jésuite au service de la position internationale du Saint-Siège}

Né en 1877, Yves de La Brière est entré dans la Compagnie en 1894. En 1909, quand Les Etudes changent de formule et renouvellent leurs collaborateurs, il y est appelé par le RP. de Grandmaison à qui elles viennent d'être confiées, pour y tenir une chronique régulière sur la "politique religieuse et internationale " 7 . Par ses origines familiales et idéologiques, La Brière est très éloigné de ces problèmes : son père était ultra-légitimiste et il ne renie jamais cette ascendance. Sa position va être d'emblée ambiguë et inconfortable. Après l'avoir convaincu d'accepter sa chronique, Grandmaison le met en garde :

"Ce que nous vous demandons de faire est très difficile et demandera parfois de vrais sacrifices. Nos amis (surtout s'ils s'occupent de politique militante) ne comprennent pas que nous n'épousions pas toutes leurs idées, toutes leurs querelles, toutes leurs préférences, toutes leurs animosités, même et surtout à l'endroit des autres catholiques qui ne pensent pas comme eux" ${ }^{8}$.

II est donc immédiatement à contreemploi, instrument de la hiérarchie pour la conversion d'un public catholique mal rallié, dont il partage les idées et les formes de pensée. Mais la logique ultramontaine contredit ses attaches nationales, et de ce hiatus sort toute sa carrière, ainsi que la dynamique de l'Eglise de France ${ }^{9}$. Dès la fin de 1915, Les Etudes, et La Brière au premier chef, sont chargés par le Pape de la propagation dans les milieux catholiques des positions internationales du SaintSiège ${ }^{10}$. En août 1915, il se fait le défenseur inlassable de Benoit XV et de ses initiatives de paix, au point de devenir son interprète autorisé. II va se trouver poussé à étudier les institutions internationales de l'après-guerre, jusqu'à en être un spécialiste reconnu dans le monde catholique français. En 1931, il résume lui-même ses "directions caractéristiques" dans un document élaboré pour sa candidature à l'Académie des Sciences Morales et Politiques :

« $1^{\circ}$ Montrer l'adaptation des principes traditionnels du christianisme... aux phénomènes internationaux de l'âge contemporain.

$2^{\circ}$ Favoriser la coopération du SaintSiège et des autres organisations catholiques aux diverses activités ou institutions présentes du monde international.

$3^{\circ}$ Faire mieux comprendre aux milieux de la droite en France que l'idée de collaboration internationale ne doit pas être considérée par eux comme suspecte ou antipathique à leurs principes, à leurs traditions, ni à leur vigilance patriotique" 11 .

C'est très consciemment qu'il s'adresse à ce public de droite, dont il est issu. Son engagement international n'infléchit en rien ses positions idéolo- 
giques et politiques, infirmant complètement la thèse reçue, selon laquelle les catholiques traditionalistes mettent leur nationalisme en avant de leur catholicisme, tandis que l'engagement international serait le fait de marginaux, venus de la "Jeune République" et des milieux démocrates-chrétiens ${ }^{12}$. S'il est vrai que la masse des catholiques reste marquée par le nationalisme, cependant on ne peut faire de La Brière un progressiste, ni un isolé. Et pourtant, il est engagé dès la Première Guerre mondiale dans le combat pour la paix par le droit.

Mgr Baudrillart, recteur de I'Institut Catholique de Paris, l'appelle, fin 1920, à la Chaire des Fondements Chrétiens du Droit des Gens, créée par la «Fondation Nationale des Morts de la Guerre" où il enseigne jusqu'en 1939. Par cette référence au droit des gens, il place son enseignement, comme son action, dans le fil des tentatives pacifistes catholiques de l'avant-guerre. II reprend en particulier l'héritage de Vanderpol, qui avait établi à Louvain une "Union Internationale pour le droit des Gens" en $1911^{13}$. Le premier niveau de son action est de définition, de médiation et de précision de l'enseignement pontifical. C'est le sujet fréquent de sa chronique aux Etudes, à commencer par celle de janvier 1915 : "La première Encyclique de Benoit XV : “Ad beatissimi»»14. II fait deux fois une visite à Rome, en 1926 et 1933 , où il soumet au Saint-Père son travail et ses directions. Celui-ci semble l'apprécier et être favorablement impressionné par son activité débordante, même s'il lui suggère de modérer son ardeur (JYLB, 2 V 1933) : en 1926, le Saint-Siège lui demande d'abandonner l'idée d'une présence officielle et permanente du Vatican à la SDN ${ }^{15}$.
Très tôt, son action n'a pas échappé aux observateurs, et L'Oeuvre lui consacre un article qui note que, dans ce cours, "la question internationale est suivie adroitement au profit des intérêts pontificaux et des doctrines catholiques. D'où nécessité de créer un enseignement équivalent et rival à la Faculté de l'Etat, sur même terrain, dans un sens nettement favorable aux conceptions laïcistes"(JYLB, 13 III 1925).

L'Institut Catholique est loin d'être le seul lieu de son enseignement. Par son état et ses fonctions, il peut profiter des structures mêmes de l'Eglise pour le diffuser. La jeunesse des écoles est particulièrement visée, celle de Paris, comme celles de Lille ou de Lyon, les trois grands foyers du catholicisme social.

Ce qui lui donne cette sorte d'autorité de spécialiste en matière internationale, c'est sa participation active à certains des mouvements internationalistes catholiques et aux activités genevoises comme il le reconnaît lui-même (JYLB, 3 X 1936).

\section{Le militant de I'UCEI}

Dans l'emploi du temps de La Brière, il est un organisme qui tient une grande place : I'Union Catholique d'Etudes Internationales. II assiste à ses réunions plusieurs fois par semaine quand il est à Paris et, lorsqu'il se rend à Genève pour assister aux sessions de la SDN, il fréquente la section suisse de l'UCEI qui est en même temps son secrétariat général. Dans ses relations, les hommes de ce mouvement tiennent la première place : le Professeur Fournier, chez qui se tiennent à Paris ces réunions, le baron de Montenach, fondateur à Fribourg en 1917 et président du mouvement, son successeur J. Reynolds. Et, surtout, Mgr Beaupin, chargé de créer en 1920 la section française du mouvement par Mgr Besson, évêque de Fribourg.

La première mention de I'UCEI dans le journal de La Brière date du 29 mai 1920, réunion préparatoire du Comité fondateur chez Fournier. Les mois suivants, les structures sont mises en place et le travail s'organise; c'est un groupe restreint de spécialistes qui travaillent en commissions séparées : coopération intellectuelle, représentation et défense des minorités, causes humanitaires, études juridiques et doctrinales. Ils se réunissent en Bureau directeur pour définir la ligne générale à suivre. II n'est pas question de vastes congrès annuels. Ainsi, les 13 et 14 novembre 1920 , pour les premières journées inter- nationales catholiques, La Brière note la présence d'une cinquantaine de participants. Puis, les 17 et 30 novembre suivants, le Bureau se réunit pour faire le point de ces journées et des premiers mois de son existence, et se posent immédiatement les problèmes de concurrence entre organismes internationaux catholiques :

«30 XI : Audace de la propagande internationale et de l'esprit d'accaparement des catholiques allemands.

Pour notre organisation, ennui de l'obstruction dirigée par Louvain contre le Centre Montenach de Fribourg... Pas commode de faire marcher les catholiques ensemble".

Ces querelles animent la vie du mouvement au début des années vingt. Elles manifestent les ambiguïtés de sa naissance. La première concurrence qui menace I'UCEI est celle de Louvain : c'est-à-dire celle de l'Union fondée par Vanderpol qui reprend ses activités en 1919. L'UCEI a donc profité de l'éclipse de Louvain pendant l'occupation allemande pour voir le jour, et elle refuse de s'effacer par la suite. Dans un premier temps, les deux organisations affirment leur désir de collaborer, mais cela n'a pas de suite. L'Union de Louvain se transforme, en novembre 1921, en «Institut de Droit International Chrétien", sous la direction d'Eugène Duthoit de Lille et de A. van den Essen de Louvain. Mgr Beaupin assiste en observateur aux séances qui réforment Louvain, où l'on convient d'un partage des tâches : à celle-ci, la réflexion et la conception des grands principes; à Fribourg, l'action et la réalisation concrètes ${ }^{16}$. La détente qui s'ensuit ${ }^{17}$ ne permet cependant pas cette répartition des tâches. Et Louvain finit par être éclipsé par Fribourg.

Les trajectoires qui mènent des catholiques à l'engagement dans les mouvements internationaux ne sont ni simples ni univoques. En général, elles viennent du catholicisme social et elles tournent autour de la confrontation avec l'Allemagne. Ainsi le chanoine Beaupin est un ancien sillonniste, ami de Marc Sangnier et aumonier de la "Jeune Garde" 18 . Appelé pendant la guerre à l' 'Office des prisonniers de guerre", il y est remarqué par Mgr Baudrillart qui lui confie le secrétariat général du "Comité des Amitiés françaises", fondé en 1915 pour contrer la propagande des catholiques allemands ${ }^{19}$, et où se retrouvent aussi Francisque Gay et Maurice Vaussard.

Pour les catholiques, comme pour les tenants d'autres universalismes, le problème que leur pose la guerre est justement celui de la préservation de cet uni- 
versalisme dans la lutte, en déniant à l'adversaire son partage. Ainsi, lors de l'occupation de la Ruhr, les Jésuites allemands des Stimmen attaquent Les Etudes pour crime de "nationalisme impérialiste", la revue française ayan trop tendance, d'après eux, à ne faire porter la responsabilité de la guerre qu'à l'Allemagne (JYLB, 19 VII 1923). Alors que la mission pontificale Testa est envoyée en Rhénanie pour observer les conditions de l'occupation française et y faire un rapport sur le respect du droit des gens, La Brière travaille à un mémoire, "sollicité" par le gouvernement français pour contrer la «propagande de Cologne", c'est-à-dire celle des catholiques rhénans. Et il porte ce mémoire au Comité des amitiés catholiques, "pour servir Patrie et Unité" (JYLB, 10 et 11 VI 1923). Si I'UCEI est un mouvement international, avec onze groupes nationaux participant au Congrès de Paris dès $1921^{20}$, en réalité elle reste essentiellement interalliée, comme le déplore le Pape d'après le Cardinal Gasparri, son Secrétaire d'Etat (JYLB, $16 \times 1925)$. Mais un an plus tard, lorsque parmi les groupes nationaux constitués se rangent l'Allemagne et l'Autriche, La Brière craint que leur participation n'entraîne leur prépondérance à Fribourg (JYLB, 14 IV 1926). II a beaucoup de mal à l'accepter sans arrièrepensée. II a beau participer régulièrement aux réunions du "Comité FranceAllemagne", surtout dans les années 1928-1929, rencontrer des "Allemands notables de haut parage" chez Wladimir d'Ormesson (JYLB, 7 ॥ 1929), assister aux messes pour la paix de Notre-Dame des Victoires, sa méfiance ne désarme pas. Quand il participe à la préparation des rencontres franco-allemandes de Berlin en décembre 1929, chez Champetier de Ribes (JYLB, 26 VI 1928, 22 XI 1929, 20 XII 1929), il note «les quelques récriminations allemandes sur le traité de Versailles", qui font convenir de laisser désormais de côté les sujets trop brûlants (JYLB, 13 XI 1930).

C'est une ligne délicate que cherchent à définir les mouvements catholiques internationaux : celle de leur internationalisme qui est leur vocation primordiale. En 1921, La Brière y revient à plusieurs reprises, en particulier en posant la double question de l'unification des organismes internationaux liés à l'Eglise et de leur participation à des rassemblements plus vastes et non confessionnels. S'il répond oui à la seconde question, sa réponse à la première est, à travers tout son journal, franchement négative, selon la ligne même de la hiérarchie comme en témoigne, cette même année, l'appréciation péjorative de $\mathrm{Mgr}$ Beaupin sur les journées de l'Internationale catholique (JYLB, 5 ॥ 1921). Et en 1925, lorsque Epstein lance son projet d'union des forces catholiques de paix au Congrès des Associations internationales catholiques à Oxford, La Brière lui oppose dans son journal "les conceptions modestes et sages de I'UCEl" (JYLB, 1 IX 1925). Reynolds écrit à Epstein sa profonde méfiance envers I'IKA, ou "Ligue internationale catholique" fondée en 1920 par l'abbé Metzger, un autrichien qui prônait cette union ${ }^{21}$. Aussi cette réunion sera-t-elle sans suite. A partir de 1927, une nouvelle tentative est lancée dans ce sens, mais cette fois-ci par l'UCEl elle-même : la "Conférence des Présidents des organisations internationales catholiques" dont la direction change chaque année. Dans une lettre à Mgr Muller, Reynolds spécifie qu'il ne s'agit pas d'une "surinternationale catholique»22. Le but de cette conférence est de former un front commun catholique sur les problèmes internationaux. Mais cette initiative ne peut qu'inquiéter le clan adverse. En septembre 1929 s'ouvrent les premières "Semaines catholiques internationales" du cercle catholique de Genève sous la direction de Mgr Besson, dont les sermons pour la paix en sa cathédrale servent de prônes officiels durant les sessions de la SDN. Mais dès 1935, le cercle catholique de Genève languit et ne remplit plus son rôle de rendez-vous international. "Pénibles désaccords et rivalités dans les milieux catholiques, une fois de plus !» (JYLB, 10 IX 1935).

\section{Le problème du recrutement des adhérents : la Ligue des Catholiques Français pour la Justice Internationale et la Paix \\ L'UCEI, pour pallier la faiblesse de} son recrutement et élargir son audience, s'adjoint, dès 1921, une Ligue qui ressuscite la "Ligue des Catholiques Français pour la Paix».

«9 IV. Question de I'UCEI et de son complément par une résurrection de l'ancienne "Société Gratry»²3, qui ferait propagande dans le public catholique pour l'organisation de la paix selon maxime chrétienne et sans utopies malfaisantes.

$10 \mathrm{~V}$. On aboutit à décider création de "La Justice Internationale".

$30 \mathrm{XI}$. Union déjà établie est un EtatMajor. Reste à créer ligue avec adhérents".
Le changement de titulature, qui élimine la "paix" au profit de la "justice" est symptomatique des changements d'état d'esprit de l'après-guerre chez les catholiques. La méfiance envers le terme même de "paix" a crû, dans le moment où, à la "Chambre Bleu Horizon", la défense religieuse est prise en charge par le "Bloc National" puis, après 1924, par la Fédération Nationale Catholique du général de Castelnau. Nous sommes toujours dans la logique du ralliement, qui suit une voie étroite entre l'éloignement des formations antirépublicaines et le refus des mouvements démocrates chrétiens. Lors de sa visite au Vatican en 1926, La Brière, qui a vraisemblablement suivi des réunions du Sillon avant guerre, et dont il dit n'avoir jamais goûté "l'atmosphère d'idéologie et d'humanitarisme" (JYLB, 21 VII 1917), "soutient les prétentions de Castelnau à la représentativité catholique" (JYLB, 22 X 1926). En pleine affaire de la condamnation de l'Action Française, ses mots montrent que l'un des aspects de l'affaire est la concurrence entre diverses formations politiques pour diriger l'opinion catholique française et que la FNC a les faveurs de la hiérarchie. Dans ces conditions, le pacifisme est encombrant. En outre, dans ces années, le public est plus sensible à la thématique de la justice qui est une réponse au problème des Réparations ${ }^{24}$. C'est un net recul, non seulement par rapport à l'avant-guerre, mais aussi par rapport aux autres mouvements européens, comme le "Friedensbund Deutschen Katholiken", qui va alors jusqu'à affirmer que toute guerre moderne est devenue illégitime ${ }^{25}$. Dès 1927 cependant, la Ligue reprend le terme de paix. L'ambiance générale a de nouveau changé : "Les catholiques français sont désormais moins exclusivement préoccupés du problème des restitutions, réparations, garanties, réclamés par la Justice Internationale", affirme Justice et Paix en $1928^{26}$. II y a une rapide conversion au briandisme ambiant du public catholique. A la suite de sa condamnation et pour contre-attaquer, l'Action Française s'est placée sur le plan international (germanophilie prétendue de la Curie), poussant les catholiques fidèles au Pape à assimiler politique vaticane et ligne briandiste alors même que Briand est un des hommes de la Séparation ${ }^{27}$.

Entre la Ligue et l'Union, les liens sont organiques. Elles ont le même siège et presque les mêmes dirigeants. E. Chénon, président de la Ligue, Mgr Beaupin, son secrétaire général, La Brière, L. 
Rolland, G. Renard, J. Douel et W. d'Ormesson sont presque tous de I'UCEI. J. Monnier voit dans le cumul des fonctions par les mêmes hommes un signe de l'étroitesse du monde catholique concerné par ces questions ${ }^{28}$. II peut s'agir aussi d'une stratégie délibérée, qui confie aux mêmes hommes les postes de direction afin de mieux tenir des mouvements sur un terrain très sensible. C'est l'Union qui a adopté les statuts de la Ligue, dont le Comité directeur est porté de 16 à 25 membres : chaque projet soumis au vote de son assemblée doit avoir été au préalable examiné par le Comité : le rôle de la base militante est bien réduit, tandis qu'à l'inverse, au niveau du Comité, chaque membre a le droit statutairement de s'expliquer avant que tout texte ne soit rédigé (JYLB, $30 \mathrm{XI}$ 1921). Est-ce pour cette méfiance vis-àvis de la base que les réunions de la Ligue deviennent très vite squelettiques selon les comptes rendus de La Brière : une vingtaine de personnes à une réunion de 1926, par exemple (JYLB, 18 XII 1926), alors que nous sommes en pleine atmosphère de Locarno, très loin encore du déclin des mouvements pacifistes : "Insouciance de la masse du public catholique français au sujet des activités internationales"? Avec la CFTC, la Ligue Catholique des femmes françaises, I'Union Féminine Civique, c'est une bonne partie du catholicisme social qui adhère à la Ligue. La Brière compte les 150000 membres de l'ACJF et les 600000 de la Ligue patriotique des Françaises comme adhérents réels de la Ligue dans l'interview qu'il accorde à la Revue Hebdomadaire 29 . Mais il ne s'agit pas d'adhésions individuelles et elles resteront toujours très fragiles. Ainsi, la "Ligue patriotique des Françaises", si enthousiaste en 1922 (JYLB, 27 I 1922), démissionne de la Ligue en novembre 1931, suivie par la "Ligue Catholique des femmes Françaises" 30. Ces démissions sont vraisemblablement provoquées par la "Déclaration des Jeunes Catholiques pour la Paix", signée par l'ACJF et par l'Action Populaire $^{31}$. En tout cas, la Ligue a manqué son but de recrutement de troupes pour I'UCEI, car elle en a adopté le fonctionnement par petits groupes de spécialistes. La conception même de leur rôle interdit en fait aux membres du Bureau de l'Union, comme à ceux de la Ligue, un recrutement plus large. Caractère qui se retrouve, Iorsqu'en 1934, La Brière définit ce que se propose l'Académie de Diplomatie internationale, à laquelle il appartient :

«Formuler un certain nombre de prin- cipes généraux dans le but de les mettre en circulation plus définis dans les milieux qualifiés. Ce qui aidera moins à obtenir des adhésions gouvernementales qu'à les faire compter comme des principes généraux du droit communément reconnu ${ }^{32}$,

Ce qui compte, c'est la qualité de quelques experts recrutés dans un souci d'efficacité, pour un travail de conversion en profondeur de la société civile et du monde politique. C'est ce qui expliquerait la très grande place que tiennent dans la vie de ces mouvements les bulletins, comme Justice et Paix, fondé par Mgr Beaupin en décembre 1922. Cette parution, irrégulière en ses origines, semble vouloir démarrer réellement fin 1928 : "Quelque vitalité reconquise» note le père de La Brière (JYLB, 4 XII 1928); elle devient mensuelle en janvier 1930, mais s'interrompt en février 1932, pour être remplacée par Informations Internationales du "Centre Catholique Français de Documentation et d'Action pour la Paix", émanation de la Ligue, créé en $1931^{33}$. La Brière est aussi en rapport avec une autre de ces publications, par l'intermédiaire de son fondateur, Maurice Vaussard, un familier du P. Sturzo, directeur de l'Ecole des Roches de 1930 à 1932, puis du Collège de Normandie de 1934 à 1938, où il invite La Brière à faire des conférences (JYLB, 26 || 1927, 17 || 1933, 16 || 1934). Vaussard avait lancé, en 1925, une "Enquête sur le Nationalisme», dans Les Lettres auprès de personnalités comme La Brière, Louis Le Fur ou Mgr Julien, évêque d'Arras, des mêmes cercles. C'est à la suite de cette enquête qu'il avait créé le Bulletin Catholique International, avec le soutien des PP. Guérin et Courtois ${ }^{34}$. Son programme et ses positions sont exactement celles de La Brière et de son groupe, bien qu'il n'y ait apparemment aucun lien institué entre eux. Ils cherchent à jouer le même rôle de "pourvoyeur d'idées dans le quadruple domaine de la lutte pour la paix, pour une nouvelle morale internationale, pour le rapprochement franco-allemand et pour la SDN»35. II est évident que, dans cette sensibilité "pacifiste", le service de la paix passe par la démonstration du caractère supranational de l'Eglise et le refus du pacifisme extrémiste. Or, c'est de ce bulletin que Marc Schérer écrit, dans la Vie Catholique, en 1938:

"ll nous avait rendus inaptes à certains sophismes du nationalisme intégral et à quelques sottises fort à la mode. II nous avait appris à aimer notre Patrie d'un coeur pur, et qu'il y avait une manière mauvaise et perverse d'aimer la Patrie qui consistait à séparer cet amour de celui de la Paix" ${ }^{36}$.

Comme pour La Brière, la portée de son discours dépasse la revue et conduit directement à des engagements qu'elle pourrait renier. Autant des mouvements plus purs et plus durs idéologiquement, comme l' «Internationale Démocratique" de Sangnier, risquent le repliement sur eux-mêmes, autant ces positions moyennes et réticentes sont grosses de toutes les évolutions possibles. C'est tout le problème des rapports entre ces mouvements et l'Action Catholique.

Le $B C l$, avec un tirage de 1500 exemplaires et 1000 abonnés, connaît rapidement des difficultés financières et sombre en décembre 1933. II est pourtant lié à l'ACJF, et a pu profiter de ses relais provinciaux vers l'opinion catholique, comme La Brière y a trouvé un auditoire plus vaste que celui de ses cours. Aux "Semaines Sociales" du Havre en 1926, devant plus de 1400 jeunes, ce dernier fait une conférence sur les structures de la SDN; autour de lui, d'autres ecclésiastiques engagés dans le même combat, les PP. Valensin, Coulet, Desbuquois, Gillet, Delos et Mgr Julien.

\section{A mi-chemin des nationalistes et des pacifistes}

II y a là, encore une fois, une profonde ambiguïté, étant données les prises de position de plus en plus pacifistes de l'Action Catholique et ses démêlées avec la droite catholique. Alors que son journal fourmille de critiques, incidentes ou plus développées des positions de Gay, de Sangnier et de tous les pacifistes démocrates chrétiens, "saugrenus et insupportables", La Brière ne cesse d'être attaqué, à partir de 1926 , par l'Action Française. A plusieurs reprises, ses conférences sont interrompues par des Camelots du Roi. Comme le lui avait prédit le $P$. de Grandmaison en 1909, sa position n'est pas bien comprise des milieux dont il est issu. Un exemple particulièrement frappant de cette distance entre le discours officiel de l'Eglise et sa réception par le public auquel il s'adresse se trouve dans un tract publié à l'occasion d'une conférence tenue en mars 1927, sous la présidence de Mgr Julien et à l'initiative de la "Fédération Française des Associations pour la SDN" ${ }^{37}$. Parmi les conférenciers, M. Aulard (Comité d'Action pour la SDN), P. de La Brière, J. Zirnheld (Président de la CFTC), de Menthon (Président de la Jeunesse catholique), $M$. 


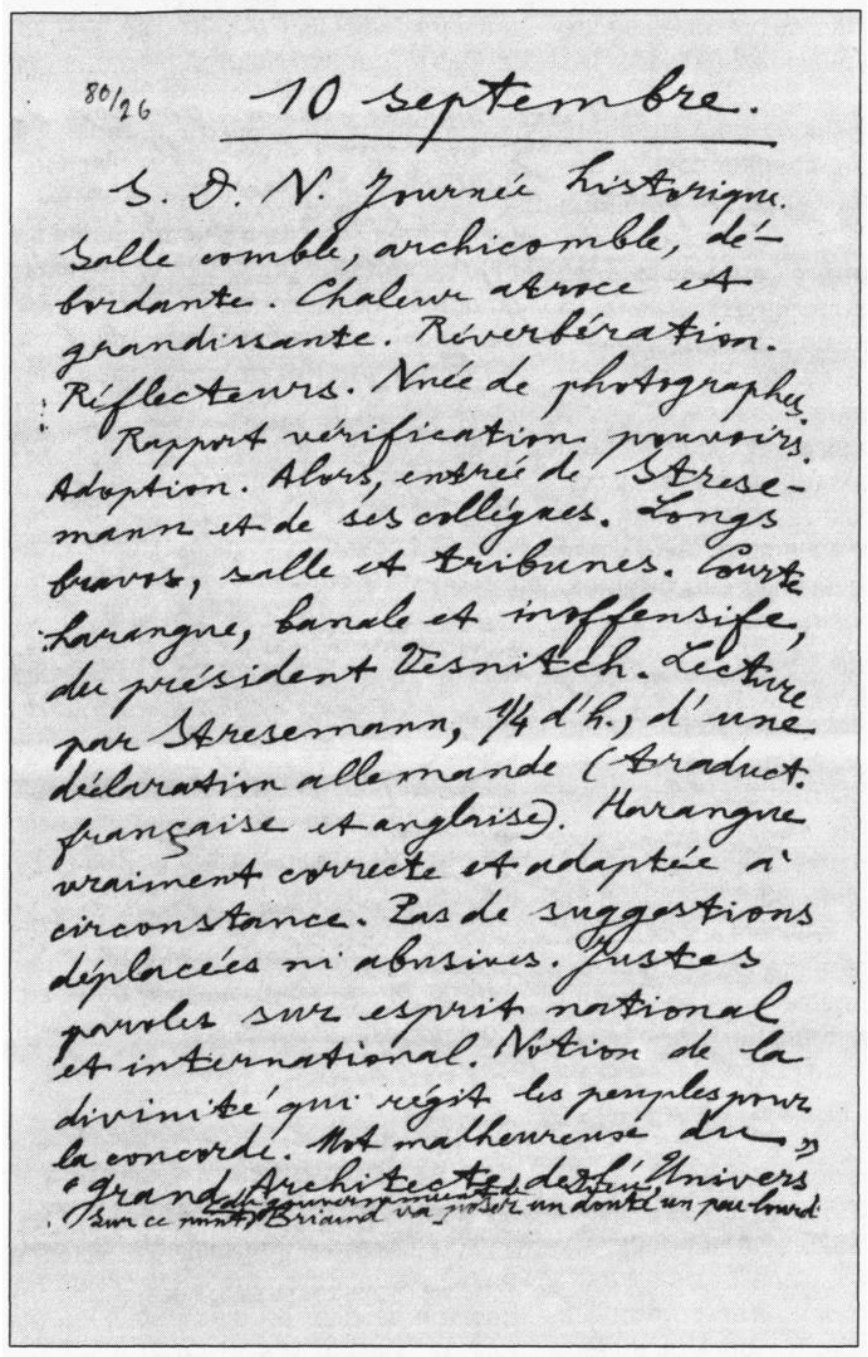

Folio 80 de l'année 1926 du journal d'Yves de La Brière, conservé à Vanves, carton 4 . Visite du Père à Genève, pour la session de la SDN où l'Allemagne est reçue.

38. Lettre de W. d'Ormesson du 21 I 1929, Papiers Lyautey. Archives nationales, $475 \mathrm{AP}$ 300.

39. Dans son numéro du 181 1931

40. Archives de la PP, Ba 1776 Dossier "Comité Catholique d'Action pour la Paix", avril 1931.

41. Voir leur liste dans $R$ Rémond, Les catholiques dans la France des années trente, Paris, Cana, 1979, nouvelle édition.
L'amalgame est plaisant, touchant des milieux qui se sont justement éloignés des excès du nationalisme maurrassien au nom de leurs conceptions aristocratiques mêmes... Dans une lettre au maréchal Lyautey, W. d'Ormesson définit ce chemin qui, d'après lui, mène du conservatisme au pacifisme :

“Car il n'y a pas au monde de force plus conservatrice que la paix. Kérillis que j'ai vu l'autre jour chez Félix de Voguë ...répétait : la guerre est volontaire, la guerre est nécessaire... je lui ai dit ce que je crois de plus en plus intensément : "voyez-vous, M. de Kérillis, entre vous et moi il y a une différence infranchissable : c'est que vous êtes un révolutionnaire et moi un conservateur» 38 .

Cette position, qui exprime celle de La Brière et de tout son milieu, montre le mécanisme qui lie le conservatisme et le pacifisme, qui les conduit à refuser le "pacifisme belliqueux". C'est toute la dynamique qui mène ces droites à l'acceptation de Munich, puis de Vichy leur pacifisme est second par rapport à leur conservatisme. Elles comprennent aussi mal la position d'un Kérillis que celle d'un Sangnier.

En 1931, Notre Temps ${ }^{39}$ publie un manifeste pacifiste, suivi en avril de la "Déclaration" que nous venons d'évoquer. La Préfecture de Police note alors que "la jeunesse catholique se montre résolument pacifiste $" 40$. La liste des organismes signataires semble conforter cette analyse ${ }^{41}$. La FNC est plus que réticente, mais la crise n'éclate entre elle et l'ACJF avec violence qu'en 1932. Depuis la guerre, celle-ci prêtait une attention toute particulière à l'enseignement pontifical sur la Paix et rejoignait ainsi La Brière. Mais surtout, elle va servir d'arme pour la reconquête de la jeunesse catholique sur l'Action Française, sous la direction de C. Flory, de 1922 à $1926^{42}$. La concurrence est directe avec Castelnau. La Brière a alors une position stratégique entre les deux camps. C'est l'ACJF qui ouvre les hostilités par un article de J. Giraud, "Au service de l'esprit de guerre", paru dans les Annales de la Jeunesse Catholique du 15 octobre 1932, où il reprend les accusations contre la collusion des EtatsMajors et des marchands de canons pendant la guerre à propos de l'affaire du bassin de Briey. Castelnau répond vivement et l'affaire s'envenime rapidement. Le 29 novembre, La Brière le reçoit et écoute ses doléances. Les deux camps font le siège du Vatican et la hiérarchie est fort embarrassée par l'affaire $^{43}$. Les Etudes prennent alors presque le rôle de Journal Officiel pour l'Eglise de France et l'on comprend mieux ce que pouvait être l'influence de La Brière. II est une des chevilles ouvrières de la propagande pontificale, qui tente alors de contrôler la presse catholique, au point que l'on a pu parler de "presse de Pie XI", avec La Croix du $P$. Merklen, avec les publications des dominicains de Juvisy ou le $\mathrm{BCl}$ de Vaussard. Les Etudes y tiennent une place de choix, par leur orthodoxie sans faille et la permanence du même chroniqueur durant tout le pontificat en la personne de La Brière ${ }^{44}$. Le 21 février 1933 , Mgr Curien, évêque de La Rochelle, se dit lors d'une visite, "fort soulagé par chroniques (de La Brière dans les Etudes) 5 janvier et 5 février. Redoutait que Vatican nous imposât ultra-pacifisme-aubiste. Motif : zèle marqué en ce sens par évêques nouvellement promus. D'où libération à voir qu'on pouvait, en toute orthodoxie, rétablir expressément la balance des choses" (JYLB, 21 II 1933).

Quand il prend la parole, en pleine affaire, à l'Ecole des Roches, La Brière consacre sa conférence aux "vrais devoirs de la paix au milieu des guerres intestines suscitées par ce concept à l'heure présente» (JYLB, 17 || 1933). C'est bien un rôle de direction et de définition du "vrai pacifisme catholique" qu'endosse le Père, et nous ne pouvons manquer de remarquer que 1926 et 1933 sont les deux dates des voyages qu'il fit à Rome, à deux moments de crise aiguë dans l'opinion publique catholique sur ces questions de nationalisme et de pacifisme. Qu'il y fût appelé pour ces questions ou qu'il y allât de luimême pour quérir des directives, en tout cas, il fait à chaque fois au Vatican un rapport sur ces questions. En 1933, il expose à Mgr Pizzardo ${ }^{45}$ les divergences entre les positions de Esprit et celles de La Vie Intellectuelle (JYLB, 2 V 1933).

La querelle rebondit en 1935 entre la FNC et les revues Septet Esprit. Dans le trouble qui s'est emparé des catholiques et que manifestent ces polémiques récurrentes, La Brière joue un rôle important, quoique discret ${ }^{46}$. Son opposition jamais désarmée à Marc Sangnier et à sa "Jeune République» est d'autant plus intéressante qu'elle ne découvre pas simplement une irréductible opposition idéologique, mais aussi une profonde divergence de stratégie militante : depuis la condamnation du Sillon, Sangnier faisait très attention à ne plus mélanger les domaines spirituels et politiques et aurait, pour cette raison, refusé 
42. P. 96 de Ch. Molette, "L'ACJF comme mouvement" in Les Mouvements de Jeunesse chrétiens et juifs : sociabilité juvénile dans un cadre européen, 1799-1968, s.d. de G. Cholvy, Paris, Le Cerf, 1985, pp. 83-108.

43. R. Rémond, op. cit.

44. M. Agostino, Le pape Pie XI et l'opinion, 1922-1939, Publication de l'Ecole Française de Rome, Paris, De Boccard, 1991, pp. 177 et S.

45. Substitut aux Affaires ecclésiastiques extraordinaires depuis 1930, il est l'un des hommes clés de la Secrétairerie d'Etat sous Pie XII.

46. R. Rémond, op. cit., p. 78

47. J. Caron, "Le Sillon et sa postéritém in Mouvements de Jeunesse, op. cit., pp. 67-74

48. E. Fouilloux, Les catho liques et l'unité chrétienne du $X I X e$ au XXe siècle. Itinéraires européens d'expression française, Paris, Le Centurion,

1982 , pp. 385 et s.

49. JYLB, 12 IV 1921, puis 14 XI 1921, 3 II 1923, 18 XI 1923. Cf. aussi R. Mayeux, Organisa tion supranationale de la paix, Paris, Ed. ouvrières, 1948, $p$ 175 qui cite YLB à propos de cette influence maçonnique.

50. S. Berstein, "Le milieu genevois de la France de l'Entre-deux-guerres" in Les Internationales, op. cit., pp. 321-335

51. Titre d'un dossier de la "Coopération intellectuelle" en 1933

52. Cf. J.-M. Mayeur in Histoire du Christianisme, op. cit., $\mathrm{p}$. 318.

53. J. Monnier, op. cit., pp 97 98. la proposition de Benoit XV de prendre la direction d'un mouvement catholique pour la paix ${ }^{47}$. La Brière a, lui, des engagements au service du Saint-Siège sans états d'âme.

\section{Un devoir de présence à la SDN et dans sa nébuleuse}

Docteur du Droit des Gens, gardien de la doctrine internationale du Saint Siège dans les mouvements catholiques, il se retrouve tout naturellement à Genève, auditeur attentif et vigilant des sessions de la SDN. II y est remarqué par ses contemporains pour son assiduité, réputation qu'il aime à rappeler (JYLB, 3 X 1936). Sa présence à Genève lui permet de fréquenter les notabilités internationales. La Brière y est comme une sorte d'ambassadeur officieux du Saint-Siège. G. Lodygensky fonde la "Commission Pro Deo" en 1933 à Genève, dans le but d'unir les forces chrétiennes en un «front unique de forces spirituelles" face au "front unique de la subversion", en clair les initiatives socialistes et bolchéviques ${ }^{48}$ Mgr Besson y délègue des adjoints et des observateurs catholiques à chaque session dont La Brière, comme en 1936 ou en 1938.

Peu auparavant, reçu à Matignon par Paul-Boncour, alors ministre d'Etat délégué permanent à Genève, avec qui il semble avoir les meilleures relations, il est poussé à aider à la création, en France, de l'équivalent de la puissante "League of Nations Union" anglaise, une sorte de confédération de tous les groupements en faveur de la SDN (JYLB, 2 IV 1936). Cette participation des catholiques à des organisations non confessionnelles est un véritable serpent de mer dans l'entre-deux-guerres. La Brière l'évoque dès 1921, sur le mode de la contre-offensive anti-maçonnique ${ }^{49}$, tout en notant le "nombre anormal de nez juifs" qui se rencontrerait dans l'Association pour la SDN créée par L. Bourgeois en 1918. Du Cartel jusqu'à l'abandon de la politique de "sécurité collective" en 1934 avec Barthou, c'est le grand moment de cette association très imprégnée de radicalisme, qui cherche à rassembler des entraîneurs d'opinion ${ }^{50}$. La Brière est l'objet de leur attention lors d'une séance à la Sorbonne en l'honneur de la cinquième assemblée de la SDN où i rencontre "un milieu où il ne fréquente pas" : Appel (recteur), Cassin, Bonnet, Jouvenel, Loucheur, Boncour, Herriot..

"Visible effort d'amabilité de leur part et satisfaction de la présence d'un représentant de l'Institut catholique dans la
Fédération universitaire». (JYLB, 24 X 1924).

La Brière est aussi un collaborateur assidu, bien que méfiant, de la «Fédération universitaire pour la SDN" et de l'«Institut de Coopération Internationale», créé par la SDN, où il est pris comme orateur du point de vue catholique à opposer à Léon Blum, pour le côté adverse (JYLB, 7 X 1924). En 1934 encore, il fait partie de la commission française de coordination des "Hautes Etudes internationales" pour le choix de sujets de concours sur le thème de la sécurité collective (JYLB, 16 | 1934). L'oeuvre d' "Entente des peuples par la jeunesse " 51 rejoint le souci de l'Eglise et donc le sien. Il revient à plusieurs reprises, pour la critiquer, sur la surveillance que certains voudraient exercer sur les manuels d'histoire afin d'en éliminer tout aspect guerrier (JYLB, 13 XI 1920, 11 VI 1924), et Mgr Beaupin luimême, un des membres catholiques de l'Institut, parle d'une éventuelle collaboration des catholiques à des «manuels raisonnables d'éducation pacifique» (JYLB, 24 IV 1926). Les catholiques jouent un rôle pondérateur face à "l'idéologie du pacifisme humanitaire qui y voltige"(JYLB, 4 IX 1925). En 1934, il fait partie du jury d'un concours sur la Paix où il joue ce même rôle pondérateur (JYLB, 3 VI 1924). La Brière est aussi en relation suivie avec "L'Ecole de la Paix» organisée par "Europe nouvelle" et Louise Weiss. En 1930, il y donne des conférences autorisées par le cardinal Verdier (JYLB, 14 X 1930), et recommence en 1931. II est alors sollicité pour rédiger une déclaration pour "la justice et la paix», à la conférence sur le désarmement tenue au Trocadéro. Dans le souci d'éviter tout "pacifisme malsain" qui menacerait cette réunion, il soumet son texte au cardinal et au père du Passage qui dirige les Etudes:

"Mon rôle est censé rester secret" (JYLB, 23-24 XI 1931).

Les destinées de I'UCEI, comme la carrière de La Brière, sont trop liées à la SDN pour ne pas en ressentir tous les avatars et les déceptions répétées. Son journal montre bien l'évolution de sa position vis-à-vis de l'institution internationale, de sa lente conversion à son utilité par delà ses origines affirmées francmaçonnes et protestantes. En 1919, il n'est question que d'un "Sur-Etat" de la SDN, "chimère de Rousseau critiquable" (JYLB, 11 III 1919); puis en 1924, de la nécessité de rompre la "solidarité injustifiée" entre la gauche et la SDN, quand certains membres du secrétariat parisien de la SDN proposent une "collaboration sympathique avec éléments de droite, avec forces catholiques pour tant d'intérêts communs ou identiques" (JYLB, 29 | 1924). Mais à partir de 1931, les constats désabusés s'accumulent. "La SDN garde-t-elle sa raison d'être ?" finit-il par se demander dans une conférence au Cercle catholique de Genève en 1934 (JYLB, 18 IX 1934), au moment où le nouveau secrétaire d'Etat, Mgr Pacelli, diplomate plus traditionnel que Mgr Gasparri, est bien moins attaché à la SDN52. Dès 1932, I'UCEI ressent le besoin de s'adapter à ce changement de situation, en dépassant le cadre des activités de la SDN et finalement ne lui survit pas ${ }^{53}$.

\section{Yves de La Brière face à la montée de la tension internationale}

C'est tout autant par une logique interne à ce pacifisme particulier que sous la pression des événements internationaux que s'explique son évolution. Les deux visites à Rome du Père de La Brière correspondent à des charnières chronologiques qui mêlent les trois niveaux, international, national et particulier. Mais dans son journal, il semble bien que les événements internationaux jouent un rôle finalement secondaire dans ses préoccupations. II fait allusion deux fois à l'Allemagne hitlérienne en 1933 (JYLB, 18 III et 16 IX 1933), et de façon plutôt neutre. En 1936, il va jusqu'à écrire à propos de l'UCEI : “Là où on ne bavarde pas sur Hitler!" (JYLB, 10 III 1936), alors que la hiérarchie condamne de plus en plus fermement le nazisme. L'exposition romaine sur la "Révolution fasciste" le laisse “oppressé et mal à l'aise» mais il précise que ce n'est pas le principe même du régime qu'il met en cause (JYLB, 23 IV 1935). Ce n'est qu'en 1936 que son ton s'altère : ses conférences s'intitulent désormais "La Papauté dans le chaos européen des jours actuels" (JYLB, 19 III 1936) ou "L'Eglise ouvrière de Paix, au milieu des peuples terrifiés par la guerre" (JYLB, 26 II 1937). II tire ce pessimisme de la session de la SDN de septembre 1935, dominée par les événements d'Ethiopie où Montenach, chef de service à la présidence de la SDN, lui décrit un ordre européen ruiné (JYLB, 19 IX 1935). Or les déceptions de la cause de la Paix qu'il énumère sont, en général, le fait des partisans des sanctions contre les coups de force allemand, italien ou espagnol, qu'il s'agisse de la remilitarisation de la Rhénanie (JYLB, 19 IX 1935) ou de façon plus globale, de 
la politique extérieure de l'Italie et de l'Allemagne:

«Discours Litvinoff pour coalition contre Etats sortis de la SDN. Argumentation qui pousse le principe de l'assistance mutuelle à des conséquences absurdes et exorbitantes, pires encore que le mal à guérir, puisque le remède serait la guerre universelle" (JYLB, 21 IX 1937).

Dans un durcissement des positions et des querelles intérieures, les tentatives de collaboration avec les mouvements non catholiques et de gauche deviennent plus difficiles. Mgr Besson partage la méfiance commune aux milieux pacifistes envers le "Rassemblement Universel pour la Paix" et refuse toute participation catholique à sa réunion prévue à Genève en 1936 (JYLB, 2 VI 1936), malgré les appels pressants de certains de ses organisateurs comme le Président de l'Union suisse pour la SDN, Bovet (JYLB, 9 VI 1936). Lorsque les représentants du RUP sont reçus par l'Assemblée de la SDN, en octobre 1936, La Brière refuse d'assister à la séance. En 1938, il est partisan d'une redistribution européenne pacifique pour régler les situations absurdes issues des traités et épouse la cause munichoise (JYLB, 23 IX 1938). Du 23 au 30 septembre, il décrit, jour après jour, les "émotions alternantes" qui font passer l'Assemblée de la SDN du pessimisme à l'optimisme. Enfin, en juin 1939, il semble devoir reprendre son travail d'explication des actes pontificaux quand il rencontre chez Daladier une certaine froideur à l'égard des initiatives conciliatrices de Pie XII (JYLB, 17 VI 1939) ${ }^{54}$. La guerre déclarée, il continue à réclamer des concessions pour la paix. Avec Wladimir d'Ormesson, il est au courant des efforts en ce sens de la part de parlementaires tels que Laval, Flandin, Bonnet, Chautemps, Piétri et il dit leur désir d'avoir le maréchal Pétain à leur tête (JYLB, $3 \mathrm{X}$ 1939). Il est ainsi étonnamment au courant de ce qui se trame dans le monde politique et il adhère à Vichy avant Vichy. En mai 1940, il est en voyage pour une tournée de conférences en Amérique du sud. II écrit de BuenosAires à sa soeur, le 5 septembre 1940, évoquant «la très noble tâche de notre gouvernement national de Vichy" 55 qui lui confie un autre cycle de conférences à faire en Amérique latine pour 1941, mais il meurt en Argentine le 25 février 1941.

Les papiers personnels d'Yves de La Brière sont précieux, car ils permettent

de voir fonctionner le mécanisme de contrôle de la hiérarchie catholique sur les organisations confessionnelles $œ u v r a n t$ pour la paix. L'Eglise est prise entre le souci d'être présente dans ce domaine pour contrer les forces laïques et ses difficultés à concevoir des structures non ecclésiales. Yves de La Brière est au coeur de la définition d'un pacifisme médian catholique et de sa mise en oeuvre. C'est tout l'intérêt de recourir aux papiers personnels d'un ecclésiastique tel que le Père de La Brière, et on aurait pu tout aussi bien étudier le très important journal d'un autre homme d'Eglise qu'il croise souvent, Monseigneur Baudrillart ${ }^{56}$. Loin d'avoir brossé un tableau complet du pacifisme catholique, nous avons pu cerner l'activité des mouvements confessionnels qui "seuls pouvaient révéler ce que fut l'action de l'Eglise catholique pour la paix, puisqu'ils dépendent entièrement de la conviction religieuse catholique, se conforment aux recommandations de l'Eglise et restent sous son contrôle hiérarchique.» 57

Bruno GOYET

Professeur agrégé d'histoire Doctorant à l'I.E.P. de Paris

Emmanuelle PICARD

Doctorante à l'I.E.P. de Paris
54. Cf. P. Blet, "Pie XII et la France en querrem, in Revue 'Histoire de l'Eglise de Fra pp. 209-232.

55. Fonds YLB, carton 2

56. II est conservé à l'Institut Catholique, sous la côte Ms 6631-6667 pour l'entre-deuxguerres.

57. J. Monnier, op. cit., pp. 4-5.

\section{Monsieur ZIRNHELI}

M. Zirnheld parle du droit des travailleurs à disposer de leur sort.

Il annonce une terrible guerre sociale en préparation. Le prochain conflit ne sera pas un conflit entre

ations, mais on conflit general entre les classes.

Ces quelques phrases de vérité sont noyées dans des dissertations sur ia Paix.

Quand il se rasseoit, le R. P. de la Brière lui serre la main avec effusion.

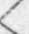

Le Père de la BRIÈRE

Le R. P. de la Brière annonce qưil ne veut donner qu'un procès verbal des rapports qu'il rédige pour

les groupenents d'études catholiques travaillant de concert avec ln Société des Nations.

II est obligé de conclure: “ Nous n'avons obtenu aucune satisfaction, mais ces rapports sont a

étnde, c'est extrèmement important:

Le R. P. de la Brière désire créer un foyer destinè a promouvoir le sens international de la jeunesse,

- Nous disposons à cet effet de très importants moyens de propagande : Toute la Jeunesse NCAISES, nous de faire pénétrer nos idées dans des centaines de mille de foyers reau.

Dans la vie de ta Justice Internationale, il faut savoir concilier lamour de ta tradition avec l'esprit nou-

\section{Madame MALATERRE-SELLIER}

- Les femmes sont trop faibles pour servir pendant la guerre, mais elles peavent mettre toute leur force morale au service des Idées internationales et être les apôtres de la Paix, dans leurs foyers, savoir Yinculque enfants en , bas âge

Cela n'empéche pas d'aimer sa patrie, mais l'amour supérieur de la patrie doit exiger des sacrifices qu'i taut savoir consentir, et, il est préférable de voir mutiler le territoire de la patrie que de laisser Elie une faute contre le dogme de linternationalisme.

Elle termine en pensant ì la satisfaction, ì la joie du cceur que doit éprouver à Rome le Saint Viellard prisonnier, à la pensée de ta, BELLE REUNION de ce soir en faveur de la paix internationale et des nobles idées qu'on y propage.

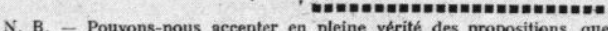

N. B. - Pouvons-nous accepter en pleine vérité des propositions que le Syllabus condamue pertinemment? Comment se defendre dun rapprochement avec les textes de différents convents maçonniques qui, dès Le poulaient liunposer au monde chrétien? Le pur bons sens appelle aussi une remarque. Puisqu'il était question de paix et quill y avait lâ un des plas rervents adeples de la guerre religieuse en la personne de M. Aulard, on attendait logiquemen Elle

Elle ne s'est pas produite, et M. Aulard n,a pas tardé bien longtemps, non seulement à proclamer

Quatre jours après le meeting, le dimanche suivant, il parlait à Saint-Dié sur la tombe de Jules erry et le journal a L Ere Nouvelle , reproduisait les paroies quil prononçait à cette occasion.

C'est un nouveau cri de guerre religieuse, ponssé cette fois contre l'Alsace-Lorraine; celle-ci ne urait rester sous le régine de tolérance et de liberté religieuse actúel et il ajoutait

" L'abrogation des lois laiques, ou en attendant cette abrogation, leur mise en sommeil " décrétée, voilà ce que recherche l'Eglise catholique, tandis qu'elle essaie d'endormir notre vigilance par son adhésion théatrale à la politique française de Paix "

Il est assez curieux également de relever dans uu livre intitulé - La Tyrannie Soviétique - Editions Spes, 1923, p. 7, écrit par Mgr d'Herbigny, grand ami du Saint-Père, le passage suivant:

: Le grand maitre pour eux (L.ENINE et ses acolytes) l'évocateur du passé, linspirateur de leurs drames - de demain. c'était M. Aulard. Depuis 1905, ses livres partaient par éditions entières pour la Russie. II " est le Pere de tout ce qui, disciplinant le Bolchevisme assura son triomphe. Contre Panarchie slave qui a'eut pu subsister, ses lectears apprirent à organiser la tyrannie. n

Quelle narveté de se fier a de tels hommes. LES CATHOLIQUEES SERONT-ILS DONC ETERNELLEMENT DUPES? 